\title{
The Association Between Prolonged Fatigue and Cardiovascular Disease in World War II Veteran Twins
}

\author{
Annette L. Fitzpatrick,' Terry Reed, ${ }^{2}$ Jack Goldberg, ${ }^{3}$ and Dedra Buchwald ${ }^{4}$ \\ 'Department of Epidemiology, University of Washington, Seattle, Washington, United States of America \\ ${ }^{2}$ Department of Medical and Molecular Genetics, Indiana University School of Medicine, Indianapolis, United States of America \\ ${ }^{3}$ Vietnam Era Twin Registry, Seattle VA Epidemiologic Research and Information Center, Seattle, Washington and Department of Epidemiology, \\ University of Washington, Seattle, Washington, United States of America \\ ${ }^{4}$ Department of Medicine, University of Washington, Seattle, Washington, United States of America
}

$\mathrm{R}$ eports of fatigue preceding cardiac events have recently been confirmed by large prospective studies. To assess for genetic confounding, we investigated prolonged fatigue and cardiovascular disease (CVD) in a cohort of World War II veteran twins. We examined data from a questionnaire mailed to members of the National Academy of Sciences-National Research Council (NAS-NRC) World War II Twins Registry in 1998 and 1999 which included questions on demographics, medical conditions and symptoms of fatigue. Data from twins discordant for prolonged fatigue lasting a month or more were analyzed using conditional logistic regression. Among 1955 twin pairs, 157 monozygotic and 174 dizygotic pairs (mean age 74 years) were discordant for prolonged fatigue. An association was found between prolonged fatigue and a history of myocardial infarction or coronary artery surgery adjusting for age, socioeconomic status, smoking, alcohol use and depression (OR [Odds Ratio]: 2.2; 95\% Cl: 1.3-4.0). When analyses were performed separately by zygosity, the association was slightly larger for monozygotic (OR: 3.3; 95\% $\mathrm{Cl}: 1.2-9.1$ ) than dizygotic twins (OR: $1.9 ; 95 \% \mathrm{Cl}$ : 0.9-4.0). These data corroborate the association of fatigue with CVD and suggest that it is not influenced by a common genetic factor. Further studies are needed to clarify the relationship and to better understand the biologic mechanisms.

Studies published in the 1970s suggested that the premonitory symptoms of cardiovascular events may be classified into three categories: chest pain, dyspnea, and fatigue (Alonzo et al., 1975; Feinleib et al., 1975; Kuller et al., 1972; Stowers \& Short, 1970). More specifically, warning symptoms prior to a myocardial infarction (MI) included tiredness as well as chest pain and breathlessness (Stowers \& Short, 1970). Others observed that fatigue and undue fatigue were among the prodromal symptoms that occurred up to 2 weeks before an MI or sudden death (Alonzo et al.,
1975; Kuller et al., 1972). Subsequently, these crosssectional associations were confirmed in prospective investigations. A study of 3877 healthy men aged between 39 and 65 years found exhaustion was associated with a greater than twofold increased risk for first MI, independent of standard risk factors such as age, blood pressure, smoking status, cholesterol, and use of antihypertensive medications (Appels et al., 2000; Appels \& Mulder, 1988). Another prospective study of 5053 college alumni also concluded that a frequent sense of exhaustion was independently associated with coronary heart disease (CHD) mortality (Cole et al., 1999). In recent studies, general feelings of malaise and lack of energy have been labeled excess fatigue (Appels \& Mulder, 1988), burn-out (Melamed et al., 1992) and vital exhaustion (Kop et al., 1994; Kopp et al., 1998). The prevalence of these symptoms, estimated to range from $30 \%$ to $60 \%$, may exceed that of any other symptom prior to cardiovascular events (Appels, 1990).

Little is known about the mechanisms that may be involved in symptoms of fatigue preceding cardiovascular events. Psychosocial roots of prolonged fatigue have been explored resulting in associations with depression and chronic stress (Cathebras et al., 1992; Lewis et al., 1994; Ware \& Kleinman, 1992). Other studies have proposed that imbalances in the pituitary-adrenal hormones, including cortisol (Keltikangas-Jarvinen et al., 1998; Nicolson \& van Diest, 2000; Raikkonen et al., 1996), blood coagulation factors (Kop et al., 1998), and inflammation (Appels, 1997a) may be responsible. In addition, several recent studies of twins have suggested that there may be a genetic susceptibility to prolonged ( 1 month or more) and chronic (6 months or more) fatigue (Buchwald et al., 2001; Hickie et al.,

Received 26 February, 2004; accepted 21 September, 2004.

Address for correspondence: Annette L. Fitzpatrick, Department of Epidemiology, University of Washington, Building 29, Suite 310, $6200 \mathrm{NE}$ 74th Street, Seattle,WA, 98115, USA.E-mail: fitzpal@u.washington.edu 
2001; Hickie et al. 1999a; Hickie et al., 1999b; Kirk et al., 1999).

We have analyzed data from 331 twin pairs in the National Academy of Sciences-National Research Council (NAS-NRC) World War II Twin Registry discordant for prolonged fatigue to examine its relationship to cardiovascular disease (CVD). The examination of fatigue using a co-twin study design provides the opportunity to control for familial and genetic effects, a strategy not previously used in studies of fatigue and CVD. In this study we investigated the following: (1) Is there an association between self-reported prolonged fatigue and CVD in a cohort of twin pairs in which one member of the pair is fatigued and the other is not?; (2) Do the associations differ between monozygotic and dizygotic twins?; and (3) Is the relationship different based on duration of the fatigue?

\section{Materials and Methods \\ Sample}

The NAS-NRC World War II Twins Registry was constructed by linking birth certificates with military record data (Jablon et al., 1967). To date, three waves of questionnaire data have been collected from the twins: between 1967 and $1978(n=14,432), 1983$ and $1986(n=9475)$, and 1998 and $1999(n=6109)$. Zygosity was assessed by a series of questions on similarity in childhood; this method has been found to be 95\% accurate compared to blood group typing and DNA analysis.

\section{Questionnaire}

The questionnaire completed by the twins in 1998 and 1999 included a wide variety of items on individual health status and behaviors including self-reported demographics (age, marital status, education), anthropometry, and self-perceived health. Socioeconomic status was based on the definition of Green (utilizing education and occupation; Green, 1970). Using selfreported height and weight information, body mass index (BMI) was calculated as ([0.45][weight in $\mathrm{lbs}] /\left[\right.$ height in $\left.\left.\mathrm{cm} / 100^{2}\right]\right)$. A man was considered to be overweight if calculated BMI was 29.5 or greater (Metropolitan Life Insurance Company, 1959). Other data collected included current use of alcohol (any amount of beer, wine or hard liquor), smoking status (current, former, never), pack-years smoked, coffee and tea intake (cups per week), blocks walked per day, and depression as measured by the Geriatric Depression Scale (Yesavage, 1988).

Information on self-reported medical conditions was collected in the questionnaire. The cardiovascular conditions of interest to our study were: MI ('heart attack'), bypass surgery/angioplasty, stroke, transient ischemic attack ('mini-stroke or transient ischemic attack'), hypertension ('high blood pressure'), and diabetes. CHD was considered to be present if a history of MI or coronary artery surgery was reported.

\section{Assessment of Fatigue}

The questionnaire also contained the following question on fatigue: 'Are you suffering from severe fatigue, extreme tiredness or exhaustion that has been present for $\geq 1$ month?' Our definition of prolonged fatigue was a positive response to this question, which corresponds to the definition developed by the Center for Disease Control and Prevention (Fukuda et al., 1994). To examine the association of a longer duration of fatigue with CVD, those twins who reported a fatigue-onset prior to 1997 were identified. Although the exact date of completing the questionnaire within the two-year data collection period was not known, this strategy guaranteed that the fatigue had been present for a minimum of one year.

\section{Statistical Analyses}

The analyses, performed on twin pairs discordant for prolonged fatigue, used a matched-pairs approach to estimate the prevalence of CVD in pairs where one twin was fatigued and other was not. Descriptive statistics were calculated as means and standard deviations for continuous variables or as numbers and percentages for categorical variables.

Conditional logistic regression, in which matching was done on twin pairs, was used to assess multivariate associations between prolonged fatigue and CVD after adjusting for risk factors. The strength of the association between prolonged fatigue and CVD was estimated by the odds ratio (OR) and associated $95 \%$ confidence interval (CI). Variables assessed for confounding in these analyses included age, marital status, education, socioeconomic status, self-perceived health, BMI, overweight status, current use of alcohol, smoking status, coffee and tea intake, activity level, and depression score. The following confounders were identified by individual assessment of variables and their impact on models of interest: socioeconomic status, depression, overweight, smoking status and use of alcohol. Models were subsequently adjusted for these variables. Hypothesis testing was conducted using Likelihood Ratio $\chi^{2}$ statistics. All data were analyzed using either SPSS for Windows (Norusis, 1993) or STATA (StataCorp, 2001).

\section{$\overline{\text { Results }}$}

A total of 6109 of $8848(69 \%)$ twins in the registry known to be alive responded to the 1998-1999 NAS-NRC Twin Registry questionnaire. Of the 1955 twin pairs for whom data were available for both members, 331 pairs were discordant for prolonged fatigue (157 monozygotic, 174 dizygotic pairs); 172 (76 monozygotic, 96 dizygotic pairs) reported onset of fatigue prior to 1997 (i.e., a year or more duration). For both the monozygotic and dizygotic pairs, demographic characteristics were similar (Table 1). Both groups had a mean age of 74 years, and over $95 \%$ had been married. They had an average of 13 years of education and a mean socioeconomic status 
Table 1

Selected Characteristics of 331 Twin Pairs Discordant for Self-Reported Prolonged Fatigue Responding to the 1998-1999 NAS-NRC WWII Veterans Twin Registry Questionnaire

\begin{tabular}{lcccc}
\hline & \multicolumn{2}{c}{$\begin{array}{c}\text { Monozygotic pairs } \\
N=157\end{array}$} & \multicolumn{2}{c}{$\begin{array}{c}\text { Dizygotic pairs } \\
N=174\end{array}$} \\
\hline Factor & Fatigued & Nonfatigued & Fatigued & Nonfatigued \\
\hline Mean age (years) & 74.6 & 74.6 & 74.2 & 74.2 \\
Ever married (\%) & 97.4 & 96.8 & 96.0 & 95.4 \\
High school graduate (\%) & 88.4 & 85.7 & 75.1 & 81.6 \\
Education (mean years) & 13.7 & 13.6 & 12.8 & 13.3 \\
Socioeconomic status (mean) & 62.7 & 62.6 & 60.4 & 61.2 \\
Weight (mean pounds) & 176.6 & 178.5 & 179.4 & 178.4 \\
Overweight (\%) & 9.9 & 14.4 & $20.1^{2}$ & 10.6 \\
\hline
\end{tabular}

Note: 'Based on Green (1970) using education and occupation.

${ }^{2}$ Chi-square $p$ testing for difference between fatigued and nonfatigued twin pairs $<.05$.

score of between 60 and 63. Although their average weight was similar (177-179 pounds), the percentage of dizygotic twins who were overweight differed by fatigue status. Over $20 \%$ of dizygotic fatigued twins were overweight compared to $10.1 \%$ of nonfatigued twins $(p<.05)$. While a greater percentage of monozygotic twins who did not report fatigue were overweight than those reporting fatigue, this difference was not significant.

Among all 331 twin pairs, the presence of prolonged fatigue was strongly associated with both previous $\mathrm{MI}(\mathrm{OR}=2.3$; 95\% CI: $1.5-3.6)$ and coronary artery surgery $(\mathrm{OR}=2.4 ; 95 \% \mathrm{CI}: 1.5-3.8$; Table 2). The combined measure of CHD generated an OR of 2.7 (95\% CI: 1.7-4.1). The strong association of fatigue with CHD remained after adjustment for age, socioeconomic, overweight, and smoking status, use of alcohol and depression (OR $=2.2 ; 95 \%$ CI: 1.3-4.0). The unadjusted association between a history of stroke and prolonged fatigue was marginally significant $(\mathrm{OR}=1.7 ; 95 \% \mathrm{CI}: 1.0-2.9)$, but after adjustment this association was attenuated $(\mathrm{OR}=1.5$;
95\% CI: 0.7-3.2). No relationships were found between prolonged fatigue and self-reported hypertension or diabetes.

The associations between prolonged fatigue and CVD were also examined by zygosity (Table 3 ). A strong association between prolonged fatigue and previous MI was observed in the monozygotic $(\mathrm{OR}=$ 4.9; 95\% CI: 1.6-14.7) but not the dizygotic twins $(\mathrm{OR}=1.2 ; 95 \% \mathrm{CI}: 0.5-2.8)$. The relationship between prolonged fatigue and previous coronary artery surgery was about twofold for both zygosity types although it was not significant in monozygotic twins. The ORs between prolonged fatigue and coronary heart disease was larger for monozygotic (OR: 3.3; 95\% CI: $1.2-0.1$ ) than dyzygotic (OR: $1.9 ; 95 \%$ CI: $0.9-4.0)$ twins, likely reflecting the larger association of previous MI in the monozygotic twins. No significant associations were found between prolonged fatigue and stroke, hypertension or diabetes when data were stratified by zygosity. Notably, point estimates for associations with CVD were similar for twins with a longer duration of fatigue although

Table 2

Association of Prolonged Fatigue and Self-Reported Cardiovascular Disease in 331 Pairs of Twins Discordant' for Fatigue in the NAS-NRC WWII Veterans Twin Registry using Conditional Logistic Regression

\begin{tabular}{lcccc}
\hline & \multicolumn{3}{c}{$\begin{array}{c}\text { Prevalence } \\
n / \text { total } N\end{array}$} & \multicolumn{2}{c}{ OR (95\% CI) } \\
\hline Factor & Fatigued & Nonfatigued & Unadjusted & Adjusted $^{2}$ \\
\hline Previous myocardial infarction & $86 / 323$ & $50 / 320$ & $2.3(1.5-3.6)$ & $2.1(1.1-3.9)$ \\
Coronary artery surgery & $95 / 324$ & $58 / 319$ & $2.4(1.5-3.8)$ & $2.1(1.1-4.1)$ \\
Coronary heart disease ${ }^{3}$ & $126 / 324$ & $76 / 320$ & $2.7(1.7-4.1)$ & $2.2(1.3-4.0)$ \\
Stroke & $51 / 321$ & $31 / 320$ & $1.7(1.0-2.9)$ & $1.5(0.7-3.2)$ \\
Hypertension & $181 / 325$ & $169 / 319$ & $1.2(0.8-1.8)$ & $1.0(0.6-1.7)$ \\
Diabetes & $83 / 324$ & $71 / 320$ & $1.3(0.8-2.0)$ & $0.9(0.4-1.7)$ \\
\hline
\end{tabular}

Note: 'One of the twin pairs responding 'yes' (the other responding 'no') to 'severe fatigue, extreme tiredness or exhaustion that has been present for $\geq 1$ month'.

${ }^{2}$ Odds ratio (confidence interval) adjusted for age, socioeconomic status, overweight, smoking status, use of alcohol, and depression.

${ }^{3}$ Either self-reported previous myocardial infarction or coronary artery surgery. 
Table 3

Association of Prolonged Fatigue and Self-Reported Cardiovascular Disease in Twins Discordant for Fatigue in the NAS-NRC WWII Veterans Twin Registry using Conditional Logistic Regression

\begin{tabular}{|c|c|c|c|c|}
\hline \multirow[b]{2}{*}{ Factor } & \multicolumn{2}{|c|}{ Fatigue $\geq 1$ Month $^{1}$} & \multicolumn{2}{|c|}{ Fatigue $\geq 1$ Year $^{2}$} \\
\hline & $\begin{array}{l}\text { Monozygotic } \\
\text { OR }(95 \% \mathrm{CI})^{3}\end{array}$ & $\begin{array}{c}\text { Dizygotic } \\
\text { OR }\left(95 \% \mathrm{Cl}^{3}\right.\end{array}$ & $\begin{array}{l}\text { Monozygotic } \\
\text { OR }\left(95 \% \mathrm{Cl}^{3}\right)^{3}\end{array}$ & $\begin{array}{c}\text { Dizygotic } \\
\text { OR }(95 \% \mathrm{CI})^{3}\end{array}$ \\
\hline Number of pairs & 157 & 174 & 76 & 96 \\
\hline Previous myocardial infarction & $4.9(1.6-14.7)$ & $1.2(0.5-2.8)$ & $4.3(1.0-18.4)$ & $1.3(0.4-3.7)$ \\
\hline Coronary artery surgery & $2.1(0.7-6.7)$ & $2.3(1.0-5.4)$ & $1.7(0.3-8.5)$ & $2.1(0.8-5.6)$ \\
\hline Coronary heart disease ${ }^{4}$ & $3.3(1.2-9.1)$ & $1.9(0.9-4.0)$ & $2.3(0.7-8.2)$ & $1.5(0.6-3.6)$ \\
\hline Stroke & $1.5(0.4-4.9)$ & $1.6(0.6-4.4)$ & $0.8(0.2-3.4)$ & $2.9(0.6-14.5)$ \\
\hline Hypertension & $1.2(0.5-2.8)$ & $0.9(0.4-1.8)$ & $0.9(0.3-2.6)$ & $1.0(0.4-2.4)$ \\
\hline Diabetes & $1.1(0.3-3.9)$ & $0.8(0.4-1.8)$ & $1.1(0.2-5.2)$ & $0.8(0.3-2.2)$ \\
\hline
\end{tabular}

Note: ' One member of the twin pair endorsing and the other denying 'severe fatigue, extreme tiredness or exhaustion that has been present for $\geq 1$ month'.

${ }^{2}$ One member of the twin pair endorsing and the other denying 'severe fatigue, extreme tiredness or exhaustion' with onset dated at or before January 1997.

${ }^{3}$ Odds ratio (confidence interval) adjusted for age, socioeconomic status, overweight, smoking status, use of alcohol, and depression.

${ }^{4}$ Either self-reported previous MI or coronary artery surgery.

fewer associations were significant due to the smaller sample size (Table 3).

\section{$\overline{\text { Discussion }}$}

Our study of 331 elderly male twin pairs derived from the NAS-NRC Twin Registry revealed a crosssectional association between prolonged fatigue of 1 month or more and self-reported coronary heart disease as defined by a history of MI or coronary artery surgery. This association persisted after adjustment for known risk factors for CHD or fatigue such as age, socioeconomic status, obesity, smoking status, use of alcohol and depression. Furthermore, the relationship was stronger in monozygotic than dizygotic twins, suggesting an absence of genetic confounding (i.e., greater genetic control did not attenuate the association). Hypertension and diabetes were not associated with prolonged fatigue.

These data corroborate the existence of a link between prolonged fatigue and CVD described in the literature. The twofold increased risk found in the adjusted model of all twins is similar in magnitude to the risks reported in prospective studies. For example, among 3877 males aged 40 to 64 participating in the Rotterdam Civil Servants Study, individuals with exhaustion, as assessed by the Maastricht Questionnaire, had twice the rate of subsequent fatal or nonfatal MI than those without exhaustion (Appels \& Mulder, 1988). Similarly, the relative risk for the association between exhaustion and death from CHD was 2.1 (95\% CI: 1.1-4.0) among 5053 male college alumni, even after adjustment for age, BMI, smoking status, obesity, and hypertension (Cole et al., 1999). Lastly, in a cross-sectional study of 3571 men, an 'imminent MI' (defined as unstable angina with electrocardiographic signs of ischemia) was greater than four times more likely to occur in exhausted and depressive persons (Appels \& Mulder, 1984). This result is similar to the association found between prolonged fatigue and previous MI in the monozygotic twins.

Excess fatigue and subclinical CVD may also be related, but results are conflicting. Subclinical cardiac decompensation, such as unrecognized ischemia or atherosclerosis, is thought to cause excess tiredness, thereby confounding the association between exhaustion and MI (Appels \& Mulder, 1988). To control for subclinical atherosclerosis, one study evaluated 127 patients who had undergone successful coronary angioplasty and observed that the presence of exhaustion two weeks postprocedure correlated with an increased risk of subsequent MIs $(\mathrm{OR}=2.7,95 \% \mathrm{CI}$ : 1.1-6.3), independent of severity of coronary artery disease and hypercholesterolemia (Kop et al., 1994). Although the authors concluded that vital exhaustion influenced the clinical course after successful coronary angioplasty in an unfavorable direction, the sample size was small and preprocedure fatigue levels were not measured. An analysis of 12,448 participants in the Atherosclerosis Risk in Communities (ARIC) cohort concluded that excess fatigue, as assessed by the Maastricht Questionnaire, and carotid intimal-medial wall thickness were not related after adjustment for other established risk factors (McGovern et al., 1996).

The available data suggests that both psychological and biological factors contribute to the association of fatigue and CVD. Along with early epidemiologic studies on the prodromata of CHD, psychiatrists investigating the mental state of coronary patients observed similar symptoms, although different terminology was used to describe them, including 'emotional drain' (Bruhn et al., 1968) and 'hidden withdrawal and masked depression' (Fischer et al., 1964). Yet evidence is emerging that depression and 
fatigue, although related, are distinct entities. In a randomly selected sample of 12,640 Hungarian adults, vital exhaustion was associated with symptoms and a history of CVD, whereas depression was more closely related to disabilities and behaviors such as alcohol/drug use, hostility, and dysfunctional cognitions (Kopp et al., 1998). Similarly, factor analysis in a cohort of 3877 men has revealed that questionnaire items assessing symptoms loaded on three unique factors reflecting dimensions of unusual fatigue, depressive affect and irritability. Moreover, fatigue had the greatest predictive power for incident MI; after controlling for fatigue, the other two dimensions lost their predictive power (Appels et al., 2000).

Efforts to understand long-term exhaustion or more rigorously defined forms of unexplained fatigue such as chronic fatigue syndrome are also of relevance here. The medical literature indicates that these conditions are not simply manifestations of underlying psychiatric disorders, but may involve autonomic dysfunction at the cardiac level as well as immunologic and infectious components (De Becker et al., 1998; Fukuda et al., 1994; Komaroff \& Buchwald, 1998; Lerner et al., 1993). In fact, an association with cardiac dysfunction and chronic fatigue has been suggested in several studies. Among 300 individuals who had worn a 24-hour Holter monitor, a significantly higher proportion of those with chronic fatigue had abnormalities consisting primarily of repetitively flat to inverted $\mathrm{T}$-waves alternating with normal $\mathrm{T}$-waves along with gross left ventricular dysfunction (Lerner et al., 1993). Others have observed that, compared to age- and sex-matched controls, patients with chronic fatigue syndrome had normal resting cardiac function but a markedly abbreviated exercise capacity characterized by slow acceleration of heart rate and fatigue of exercising muscles long before peak heart rate was achieved (Montague et al., 1989).

Other investigations into the mechanisms underlying the link between prolonged fatigue and CVD have implicated pituitary adrenal hormones, primarily cortisol (Keltikangas-Jarvinen et al., 1998; Nicolson \& van Diest, 2000; Raikkonen et al., 1996). One author has hypothesized that the decreased activity of the hypothalamic-pituitary-adrenal axis may reflect a homeostatic reaction to prolonged stress and inflammation and, therefore, treatment of these conditions may reduce cardiac events (Appels, 1997a; Appels, 1997b). In this regard, basal salivary cortisol levels were lower in individuals with vital exhaustion than in controls in cross-sectional studies (Nicolson \& van Diest, 2000). Feelings of exhaustion and emotional distress have also been associated with lowered hypothalamic-pituitary-adrenal activity; this activity was able to distinguish borderline hypertensives from normotensives adjusting for other risk factors (Raikkonen et al., 1996). Vital exhaustion was also associated with significantly elevated levels of plasminogen activator inhibitor (PAI-1) activity after controlling for smoking status and serum lipids suggesting that reduced fibrinolytic capacity may mediate the relationship between vital exhaustion and the risk of MI (Kop et al., 1998).

Of note, a dose-response relationship between duration of fatigue and heart disease was not found although this may have been affected by our inability to assess specific lengths of duration. Others have reported the association between fatigue and coronary heart disease to be strongest most proximal to the measurement of the fatigue (Appels \& Otten, 1992). In a cohort of 3365 males ages 45 to 59 years subsequently followed for an average of 9.2 years, the hazard ratios for associations between exhaustion and cardiac death were 9.0, 6.3, 4.5 and 3.2 for the first 10, 20, 30 and 40 months of follow-up respectively. After 40 months, the association was no longer significant. This data suggests that a cardiovascular event is more likely to occur after new onset of symptoms rather than after fatigue has been present for many years.

A unique feature of our investigation that deserves mention is the use of fatigue in discordant twins. This study design is used to provide evidence of a common genetic vulnerability for two or more traits when an association is found (Kendler et al., 1993). It is assumed that the phenotypic association will disappear when genotype is controlled. Several recent studies of twins have suggested that there may be a genetic susceptibility to prolonged ( $\geq 1$ month) and chronic ( $\geq 6$ months) fatigue (Buchwald et al., 2001; Hickie et al., 2001; Hickie et al., 1999a; Hickie et al., 1999b; Kirk et al., 1999). Interestingly, several of these papers also found that the genetic and environmental influences on fatigue were, for the most part, independent of those for anxiety, depression and psychological distress (Hickie et al., 1999a; Hickie et al., 1999b; Kirk et al., 1999). Twin studies have also suggested that in addition to genetic influences, immune responsiveness may be environmentally related to fatigue (Hickie et al., 2001; Hickie et al., 1999a). This study did not suggest the involvement of genetic factors in the association between prolonged fatigue and CVD as the elevated risks remained in both the mono- and dizygotic twins, and the risk was not larger in the dizygotes. This is important as it may indicate that fatigue related to prodromata of CVD is of a different etiology than that involved in immune dysfunction and symptoms of chronic fatigue syndrome.

This study has several limitations. First, its crosssectional design did not allow the assessment of the temporal relationship of fatigue and CVD, that is, we could not separate fatigue preceding from that following MI and coronary artery surgery. Others, however, have demonstrated in prospective studies that these symptoms do indeed precede cardiac events. The primary contribution with these analyses was the assessment of genetic confounding and not assessment of temporality of the association. Second, CVD is very likely understated here as only more severe cases result- 
ing in clinical symptoms of $\mathrm{MI}$ and those indicative of surgery, were reported. Misclassification may also have occurred through self-report of diseases and fatigue. Although the definition of prolonged fatigue was based on that of the Centers for Disease Control and Prevention (Fukuda et al., 1994), the perception of 'severe fatigue, extreme tiredness or exhaustion' may vary considerably between individuals. Finally, the impact of nonresponse in this survey is unclear since both men with fatigue and/or CVD may have been less likely to respond. Nonetheless, the prevalence of diagnosed CVD in the sample approximated that in the general US male population (American Heart Association, 2002). Regardless of these limitations, this co-twin study contributes to the literature on fatigue and CVD by controlling for genetic influences to a greater extent than was possible in previous studies.

In summary, the data collected from the NAS-NRC WWII Twin Registry corroborated the previously reported relationship between prolonged fatigue and coronary heart disease. The findings indicate that genetic confounding is not involved and that the mediating factor is not a CVD-related risk factor such as hypertension or diabetes. These data underscore the importance of fatigue as a possible precursor to cardiac events. This information could be useful in clinical settings, particularly since fatigue is not typically considered a cardinal symptom of CVD, to identify individuals potentially at high risk. Cohort studies are needed to clarify the temporal relationship between prolonged fatigue and coronary heart disease to better understand the biologic mechanism, and to reduce the morbidity and mortality of CVD.

\section{Acknowledgment}

This work was funded by grant U19 AI38429 from the National Institutes of Health (Drs. Goldberg and Buchwald).

\section{References}

Alonzo, A., Simon, A., \& Feinleib, M. (1975). Prodromata of myocardial infarction and sudden death. Circulation, 52, 1056-1062.

American Heart Association. (2002). Heart Disease and Stroke Statistics: 2002 Update. Dallas, TX: American Heart Association.

Appels, A. (1990). Mental precursors of myocardial infarction. British Journal of Psychiatry, 156, 465-471.

Appels, A. (1997a). Exhausted subjects, exhausted systems. Acta Physiologica Scandinavica, 640(Suppl.), 153-154.

Appels, A. (1997b). Why do imminent victims of a cardiac event feel so tired? International Journal of Clinical Practice, 7, 447-450.

Appels, A., Kop, W. J., \& Schouten, E. (2000). The nature of the depressive symptomatology preceding myocardial infarction. Behavioral Medicine, 26, 86-89.

Appels, A., \& Mulder, P. (1984). Imminent myocardial infarction: A psychological study. Journal of Human Stress, 10, 129-134.

Appels, A., \& Mulder, P. (1988). Excess fatigue as a precurser of myocardial infarction. European Heart Journal, 9, 758-764.

Appels, A., \& Otten, F. (1992). Exhaustion as a precurser of cardiac death. British Journal of Clinical Psychology, 31, 51-56.

Bruhn, J. G., McCrady, K., \& Plesis, A. (1968). Evidence of 'emotional drain' preceding death from myocardial infarction. Psychiatry Digest, 29, 34-40.

Buchwald, D., Herrell, R., Ashton, S., Belcourt, M., Schmaling, K., Sullivan, P., Neale, M., \& Goldberg, J. (2001). A twin study of chronic fatigue. Psychosomatic Medicine, 63, 936-943.

Cathebras, P. G., Robbins, J. M., Kinmayer, L. J., \& Hayton, B. C. (1992). Fatigue in primary care: Prevalence, psychiatric comorbidity, illness behavior and outcome. Journal of General Internal Medicine, 7, 276-286.

Cole, S. R., Kawachi, I., Sesso, H. D., Paffenbarger, R. S., \& Lee, I. M. (1999). Sense of exhaustion and coronary heart disease among college alumni. American Journal of Cardiology, 84, 1401-1405.

De Becker, P., Dendale, P., De Meileir, K., Meirleir, K., Campine, I., Vandenborne, K., \& Hagers, Y. (1998). Autonomic testing in patients with chronic fatigue syndrome. American Journal of Medicine, 105, 22S-26S.

Feinleib, M., Simon, A., Gillium, R., \& Margolis, J. R. (1975). Prodromal symptoms and signs of sudden death. Circulation, 52(Suppl. III), 155-159.

Fischer, H. K., Dlin, B. M., Winters, W. L., Jr., Hagner, S. B., Russell, G. W., \& Weiss, E. (1964). Emotional factors in coronary occlusion II: Time patterns and factors related to onset. Pschosomatics, 5, 280-291.

Fukuda, K., Straus, S. E., Hickie, I., Sharpe, M. C., Dobbins, J. G., \& Komaroff, A. (1994). The chronic fatigue syndrome: A comprehensive approach to its definition and study. Annals of Internal Medicine, 121, 953-959.

Green, L. W. (1970). Manual for scoring socioeconomic status for research and behavior. Public Health Report, 85, 815-827.

Hickie, I. B., Bansal, A. S., Kirk, K. M., Lloyd, A. R., \& Martin, N. G. (2001). A twin study of the etiology of prolonged fatigue and immune activation. Twin Research, 4, 94-102.

Hickie, I., Bennett, B., Lloyd, A., Heath, A., \& Martin, N. (1999a). Complex genetic and environmental relationships between psychological distress, fatigue and immune functioning: a twin study. Psychological Medicine, 29, 269-277. 
Hickie, I. B., Kirk, K., \& Martin, N. (1999b). Unique genetic and environmental determinants of prolonged fatigue: A twin study. Psychological Medicine, 29, 259-268.

Jablon, S., Neel, J. V., Gershowitz, H., \& Atkinson, G. F. (1967). The NAS-NRC twin panel: Methods of construction of the panel, zygosity diagnosis and proposed use. American Journal of Human Genetics, 19, 133-161.

Keltikangas-Jarvinen, L., Ravaja, N., Raikkonen, K., Hautanen, A., \& Adlercreutz, H. (1998). Relationships between pituitary-adrenal hormones, insulin, and glucose in middle-aged men: Moderating influence of psychosocial stress. Metabolism, 47, 1440-1449.

Kendler, K. S., Neale, M. C., MacLean, C. J., Heath, A. C., Eaves L. J., \& Kessler, R. C. (1993). Smoking and major depression. A causal analysis. Archives of General Psychiatry, 50, 36-43.

Kirk, K. M., Hickie, I. B., \& Martin, N. G. (1999). Fatigue as related to anxiety and depression in a community-based sample of twins aged over 50. Social Psychiatry Epidemiology, 34, 85-90.

Komaroff, A. L., \& Buchwald, D. S. (1998). Chronic fatigue syndrome: An update. Annual Review of Medicine, 49, 1-13.

Kop, W. J., Appels, A., Mendes de Leon, C. F., Swart, H. B., \& Bar, F. W. (1994). Vital exhaustion predicts new cardiac events after successful coronary angioplasty. Psychosomatic Medicine, 56, 281-287.

Kop, W. J., Hamulyak, K., Pernot, C., \& Appels, A. (1998). Relationship of blood coagulation and fibrinolysis to vital exhaustion. Psychosomatic Medicine, 60, 352-358.

Kopp, M. S., Falger, P. R. J., Appels, A., \& Szedmak, S. (1998). Depressive symptomatology and vital exhaustion are differentially related to behavioral risk factors for coronary artery disease. Psychosomatic Medicine, 60, 752-758.

Kuller, L. K., Cooper, M., \& Perper, J. (1972). Epidemiology of sudden death. Archives of Internal Medicine, 129, 714-719.

Lerner, A. M., Lawrie, C., \& Dworkin, H. S. (1993). Repetitively negative changing $\mathrm{T}$ waves at 24-h electrocariographic monitors in patients with the chronic fatigue syndrome: Left ventricular dysfunction in a cohort. Chest, 104, 1417-1421.

Lewis, S., Cooper, C. L., \& Bennett, D. (1994). Psychosocial factors and chronic fatigue syndrome. Psychological Medicine, 13, 661-671.

McGovern, P. G., Shahar, E., Folsom, A. R., \& Rosamond, W. D. (1996). No relation between excess fatigue and asymptomatic carotid arteriosclerosis. Epidemiology, 6, 638-640.

Melamed, S., Kushnir, T., \& Shirom, A. (1992). Burnout and risk factors for cardiovascular disease. Behavioral Medicine, 18, 53-60.

Metropolitan Life Insurance Company. (1959). New weight standards for men and women. Statistical Bulletin of the Metropolitan Life Insurance Company, 40, 1-4.

Montague, T. J., Marrie, T. J., Klassen, G. A., Bewick, D. J., \& Horacek, B. M. (1989). Cardiac function at rest and with exercise in the chronic fatigue syndrome. Chest, 95, 779-784.

Nicolson, N. A., \& van Diest, R. (2000). Salivary cortisol patterns in vital exhaustion. Journal of Psychosomatic Research, 49, 335-342.

Norusis, M. J. (1993). SPSS for Windows, advanced statistics (Release 6.0) [Computer software]. Chicago, IL: SPSS.

Raikkonen, K., Huatanen, A., \& Keltikangas-Jarvinen, L. (1996). Feelings of exhaustion, emotional distress, and pituitary and adrenocortical hormones in borderline hypertension. Journal of Hypertension, 14, 713-718.

StataCorp. (2001). Stata statistical software (Release 7.0) [Computer software]. College Station, TX: Stata Corporation.

Stowers, M., \& Short, D. (1970). Warning symptoms before myocardial infarction. British Heart Journal, 32, 833-838.

Ware, N. C., \& Kleinman, A. (1992). Culture and somatic experience: The social course of illness in neurasthenia and chronic fatigue syndrome. Psychosomatic Medicine, 54, 546-560.

Yesavage, J. A. (1988). Geriatric depression scale. Psychopharmacology Bulletin, 24, 709-710. 